Research, Society and Development, v. 9, n. 10, e5649108846, 2020

(CC BY 4.0) | ISSN 2525-3409 | DOI: http://dx.doi.org/10.33448/rsd-v9i10.8846

\title{
Tratamento videolaparoscópico para neoplasia de cólon direito: relato de caso
}

Videolaparoscopic treatment for right colon neoplasia: case report

Tratamiento videolaparoscópico de la neoplasia del colon derecho: reporte de caso

Recebido: 27/09/2020 | Revisado: 28/10/2020 | Aceito: 07/10/2020 | Publicado: 08/10/2020

\section{Marcela Araujo Pereira}

ORCID: https://orcid.org/0000-0002-8377-7610

Centro Universitário Tocantinense Presidente Antônio Carlos, Brasil

E-mail:marcelaacruvinel@gmail.com

Rone Antônio Alves de Abreu

ORCID: https://orcid.org/0000-0002-7821-2463

Centro Universitário Tocantinense Presidente Antônio Carlos, Brasil

E-mail: roneantonioabreu@gmail.com

Rebeca Fontoura Morais Abreu

ORCID: https://orcid.org/0000-0001-9932-0861

Centro Universitário Tocantinense Presidente Antônio Carlos, Brasil

E-mail: rebeca.fontoura@hotmail.com

Rone Fontoura Abreu

ORCID: https://orcid.org/0000-0002-7595-5659

Centro Universitário Tocantinense Presidente Antônio Carlos, Brasil

E-mail: ronefontoura@hotmail.com

Gabriela Vellano de Andrade

ORCID: https://orcid.org/0000-0003-2519-5337

Centro Universitário Tocantinense Presidente Antônio Carlos, Brasil

E-mail: gabivellano@gmail.com

Fabiana Gallo

ORCID: https://orcid.org/0000-0002-8526-1538

Centro Universitário Tocantinense Presidente Antônio Carlos, Brasil

E-mail: fabiana-gallo@hotmail.com

Pedro Ernesto Alves Mangueira Júnior

ORCID: https://orcid.org/0000-0003-2976-7443

Centro Universitário Tocantinense Presidente Antônio Carlos, Brasil

E-mail: pedromangueirajr@gmail.com 


\title{
Resumo
}

Objetivo: relatar um caso de neoplasia de cólon direito em paciente submetido a hemicolectomia laparoscópica no Hospital e Maternidade Dom Orione (HMDO), no estado do Tocantins e discutir seus aspectos clínicos, evolutivos, diagnósticos e terapêuticos, ressaltando a importância do diagnóstico precoce para evitar a progressão da doença. Métodos: Apresenta-se um caso de hemicolectomia direitalaparoscópica em paciente portador de neoplasia de cólon ascendente caracterizando um estudo retrospectivo. Para isto foram utilizados os prontuários localizados no Hospital e Maternidade Dom Orione e da Clínica Gastromed. Resultados: Paciente masculino, 54 anos, procurou a equipe de saúde referindo dor abdominal, moderada em hipocôndrio direito, em cólica, insidiosa, sem irradiação, associada à alteração do hábito intestinal e anemia. Antecedentes: hipertensão arterial, diabetes melito e infarto agudo do miocárdio. Feita a hipótese de neoplasia de cólon, solicitou-se colonoscopia que corroborou com a hipótese inicial. A biópsia identificou um adenocarcinoma tubular moderadamente diferenciado. Os exames laboratoriais se mostravam normais, a não ser pela presença de anemia microcítica e hipocrômica. Paciente submetido a estadiamento: TC de torax e abdome total sem evidências de metástases. CEA: 48,1 (até 3,8 ng/mL). Após ser avaliado pela Cirurgia Oncológica, foi indicado Hemicolectomia direita laparoscópica com linfadenectomia, ileotransverso-anastomose látero-lateral e drenagem da cavidade. Conclusão: Nas condições que foram realizados o presente estudo podemos concluir que a via lapaoscópica se mostrou como um método eficaz para o tratamento deste paciente portador de neoplasia de cólon ascendente.

Palavras-Chave: Neoplasia colorretal; Hemicolectomia; Laparoscopia; Colonoscopia; Cólon direito; Adenocarcinoma.

\begin{abstract}
Objectives: to report a case of right colon cancer in a patient undergoing laparoscopic hemicolectomy at Hospital and Maternidade Dom Orione (HMDO), in the state of Tocantins and to discuss its clinical, evolutionary, diagnostic and therapeutic aspects, emphasizing the importance of early diagnosis to avoid disease progression. Methods: A case of right-side paraoscopic hemicolectomy is presented in a patient with ascending colon neoplasia, characterizing a retrospective study. For this purpose, the medical records located at Dom Orione Hospital and Maternity Hospital and Clínica Gastromed were used. Results: Male patient, 54 years old, went to the health team, reporting abdominal pain, moderate in the right hypochondrium, colic, insidious, without irradiation, associated with changes in bowel habits
\end{abstract}


and anemia. Background: arterial hypertension, diabetes mellitus and acute myocardial infarction. After the hypothesis of colon neoplasia, colonoscopy was requested, which corroborated the initial hypothesis. The biopsy identified a moderately differentiated tubular adenocarcinoma. Laboratory tests were normal, except for the presence of microcytic and hypochromic anemia. Patient undergoing staging: CT scan of the chest and total abdomen without evidence of metastasis. CEA: 48.1 (up to $3.8 \mathrm{ng} / \mathrm{mL}$ ). After being evaluated by Oncological Surgery, laparoscopic right hemicolectomy with lymphadenectomy, ileotransversion-lateral-lateral anastomosis and drainage of the cavity was indicated. Conclusion: Under the conditions that were carried out in the present study, we can conclude that the lapaoscopic route proved to be an effective method for the treatment of this patient with ascending colon neoplasia.

Keywords: Colorectal neoplasia; Hemicolectomy; Laparoscopy; Colonoscopy; Right colon; Adenocarcinoma.

\section{Resumem}

Objetivos: reportar un caso de cáncer de colon derecho en un paciente sometido a hemicolectomía laparoscópica en el Hospital y Maternidade Dom Orione (HMDO), en el estado de Tocantins y discutir sus aspectos clínicos, evolutivos, diagnósticos y terapéuticos, enfatizando la importancia del diagnóstico precoz para evitar enfermedad progresiva. Métodos: Se presenta un caso de hemicolectomía paraoscópica derecha en un paciente con neoplasia de colon ascendente, caracterizando un estudio retrospectivo. Para ello se utilizaron las historias clínicas ubicadas en el Hospital y Maternidad Dom Orione y Clínica Gastromed. Resultados: Paciente masculino de 54 años que acudió al equipo de salud, refiriendo dolor abdominal moderado en hipocondrio derecho, cólico, insidioso, sin irradiación, asociado a cambios en los hábitos intestinales y anemia. Antecedentes: hipertensión arterial, diabetes mellitus e infarto agudo de miocardio. Tras la hipótesis de neoplasia de colon, se solicitó una colonoscopia, que corroboró la hipótesis inicial. La biopsia identificó un adenocarcinoma tubular moderadamente diferenciado. Los análisis de laboratorio fueron normales, excepto por la presencia de anemia microcítica e hipocrómica. Paciente sometido a estadificación: TAC de tórax y abdomen total sin evidencia de metástasis. CEA: 48,1 (hasta 3,8 $\mathrm{ng} / \mathrm{mL}$ ). Tras ser evaluado por Cirugía Oncológica, se indicó hemicolectomía derecha laparoscópica con linfadenectomía, anastomosis ileotransversión-lateral-lateral y drenaje de la cavidad. Conclusión: En las condiciones que se llevaron a cabo en el presente estudio, podemos 
concluir que la vía lapaoscópica resultó ser un método eficaz para el tratamiento de este paciente con neoplasia de colon ascendente.

Palabras clave: Neoplasia colorrectal; Hemicolectomía; Laparoscopia; Colonoscopia; Colon derecho; Adenocarcinoma.

\section{Introdução}

A neoplasia maligna de cólon é o câncer mais frequente do trato gastrointestinal. Propõe-se que 36.360 pessoas serão diagnosticadas com câncer de cólon e reto em 2018. No Tocantins, a estimativa de casos novos para 2018 é de 50 dentre os homens e 60 dentre as mulheres. Em relação a mortalidade, a neoplasia maligna colorretal corresponde à quarta causa de morte por câncer no sexo masculino e, à terceira no sexo feminino (INCA, 2018).

Na maioria dos casos o câncer colorretal é adenocarcinoma, derivado do epitélio glandular colônico. Em termos de localização, houve uma inversão recente da incidência, que passou a predominar no colo direito. Muitos estudos revelam maiores taxas de sobrevida para estes, com piora do prognóstico a medida que as lesões se situam mais distalmente ()

Dispepsias, sensação de mal-estar, melena e fadiga são sintomas comuns de neoplasia de cólon direito. Enquanto, sintomas obstrutivos e alteração dos hábitos intestinais falam mais a favor da topografia a esquerda. O diagnóstico pode ser investigado a partir de exames de triagem como, exame digital retal, sangue oculto nas fezes, retossigmoideoscopia, enema opaco, permanecendo como padrão ouro a colonoscopia que permite o diagnóstico através da biopsia de lesões suspeitas ()

A cirurgia continua sendo o tratamento mais adequado para o câncer colorretal, tornando possível a cura em grande parte dos casos. Sendo assim, hemicolectomia associada a linfadenectomia o procedimento de escolha para estes pacientes. Dessa forma, a melhor compreensão da patologia, poderá permitir um diagnóstico precoce, evitando a evolução desta e o desenvolvimento de complicações, melhorando assim o prognóstico ().

Tendo tipo a oportunidade de tratar um paciente portador de neoplasia de cólon direito por via laparoscópica achamos interessante apresentar o presente relato. 


\section{Métodos}

\subsection{A natureza da pesquisa}

Trata-se de um estudo de caso, retrospectivo e descritivo com abordagem qualitativa. Como cita Pereira et al. (2018), um estudo de caso é uma descrição e análise o mais detalhada possível de algum caso e caracteriza-se qualitativo quando é importante a interpretação do pesquisador e suas opiniões sobre o fenômeno relatado, tendendo a seguir um processo indutivo. Sendo assim, este estudo irá abordar o caso de um paciente com neoplasia de cólon direito, utilizando para isso o prontuário do paciente em questão após aprovação do projeto pelo CEP e da autorização por escrito do paciente e da instituição em que será realizada a coleta dos dados. Será realizada durante todo o período de estudo consultas a literatura disponível e pertinente ao assunto, tais como: artigos científicos, livros, revistas, etc.

\subsection{O universo e o local da pesquisa}

A pesquisa será realizada na clínica Gastromed e no Hospital e Maternidade Dom Orione, ambos situados em Araguaína- TO. A escolha do local se deve a facilidade do acesso aos prontuários e ao paciente.

\subsection{Instrumentos de coleta de dados}

Para coleta de dados será utilizada análise do prontuário, demonstrando a atuação do médico cirurgião diante das novidades terapêuticas para esta frequente patologia com escassos estudos comparando esta atuação com a literatura disponível.

Serão analisados os exames de imagem disponíveis junto ao prontuário do paciente juntamente com os métodos terapêuticos empregados no tratamento da patologia.

\subsection{Instrumento de coleta de dados}

Os dados serão coletados durante a análise do prontuário e depois serão transcritos todos os dados para documento Word em caráter cronológico e seguindo as normas da Associação Brasileira de Normas Técnicas (ABNT). Antes do início do trabalho, o paciente será convidado a participar do mesmo e para isso todas as informações referentes ao estudo 
lhe serão explicadas por meio do termo de consentimento livre e esclarecido de acordo com a resolução do CONEP 466/2012. O projeto de pesquisa passará por várias etapas: seleção do tópico ou problema para investigação, definição e diferenciação do problema, levantamento de hipóteses, coleta, sistematização e classificação dos dados, análise e interpretação dos dados e relatório do resultado de pesquisa.

\subsection{Análise dos dados}

O estudo de caso será precedido pela exposição do problema com questões orientadoras, que compõem a teoria preliminar que será avaliada à partir dos achados da pesquisa, de uma plataforma teórica, de um detalhado planejamento de toda a investigação, destacando-se a construção de um protocolo do caso, contendo descrição dos instrumentos de coleta de dados e evidências, estratégias de coleta e análises dos dados, possíveis triangulações de dados, prováveis encadeamentos de evidências e avaliações da teoria previamente admitida, com afinidade de se construir uma teoria para explicação do objeto de estudo: o caso. Após a coleta, os dados serão transcritos e discutidos. A pesquisa bibliográfica será realizada utilizando os bancos de dados MEDLINE, LILACS-BIREME, PUBMED e COCHRANE, além de livros e revistas que contenham o assunto pertinente ao trabalho e que tenham sido publicados nos últimos dez anos. Os seguintes termos de pesquisa (palavraschaves e delimitadores) serão utilizados em várias combinações: 1) neoplasia colorretal ; 2) adenocarcionoma de cólon direito; 3) hemicolectomia; 4) laparoscopia oncológica. A pesquisa bibliográfica também inclui livros e periódicos analisados na biblioteca do Instituto Tocantinense Presidente Antônio Carlos.

\section{Resultados}

JPS, 54 anos, diabético, hipertenso, no dia 11/04, procurou a equipe de saúde referindo dor abdominal moderada em hipocôndrio direito, insidiosa, sem irradiação, associada à alteração do hábito intestinal e anemia crônica. Paciente relatou infarto agudo do miocárdio de ramo marginal esquerdo, em 2012, com realização de tratamento adequado. Faz uso regular de concardio, metformina, losartana e espironolactona. Nega alergias medicamentosas. Nega emagrecimento. 
Figura 1. Colonoscopia evidenciando lesão estenótica no cólon ascendente.

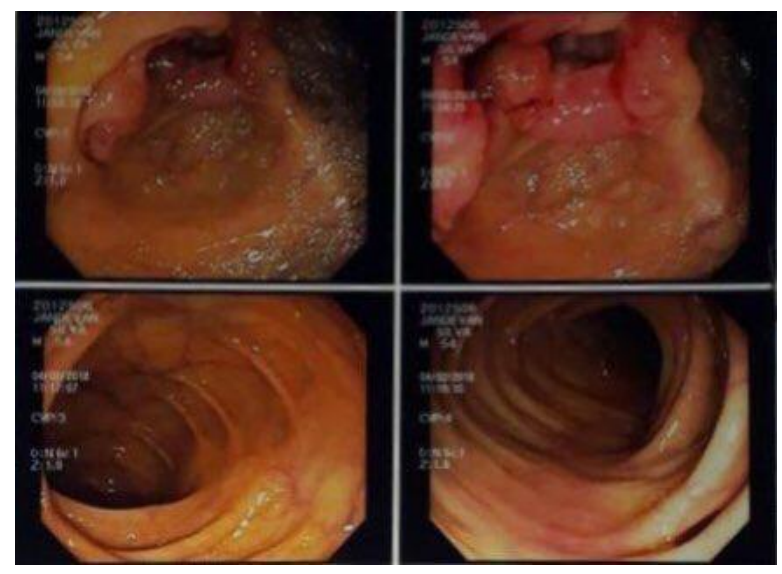

Fonte: Arquivo Pessoal

Feita a hipótese de neoplasia de cólon, foi solicitado uma colonoscopia, que evidenciou: no cólon ascendente, uma extensa lesão vegetante, com formato irregular, multinodular ou multibocelada (Figura 1), consistência endurecida e fácil sangramento, com dificuldade de progressão do colonoscópio, corroborando com a hipótese diagnóstica principal; e reto, cólon descendente e cólon transverso com aspecto normal e óstios diverticulares de pequeno tamanho no sigmoide. A biópsia realizada identificou um adenocarcinoma tubular moderadamente diferenciado.

Hemoglobina: 9,4 mg/dL; Hematócrito: 32,6\%; Leucócitos: 5.800 mm3; Neutófilos: 4.176 mm3; Glicemia: 135 mg/dL; Tempo de Sangramento (TS): 1min e 30 seg; Tempo de Coagulação (TeC): 6min; Plaquetas: 385.000 mm3 ; Prova do laço negativa; Ureia: $34 \mathrm{mg} / \mathrm{dL}$; Creatinina: 1,02 mg/dL; Proteínas totais: 6,9 g/dL; Albumina 4,6 g/dL; Globulina 2,3 g/dL; Relação A/G: 2; Sódio: $141 \mathrm{mmol} / \mathrm{L}$; Potásio: 4,7 mmol/L; Antígeno Carcinoembriogênico (CEA): $48,47 \mathrm{ng} / \mathrm{mL}$.

Figura 2. (A) TC de tórax, janela de pulmão sem evidências de acometimento do parênquima; (B) TC de abdome total: fígado, com morfologia, dimensões e coeficiente de atenuação normais.- afastando metástases à distância; (C) TC de abdome total: lesão no cólon direito sugestiva de neoplasia com acometimento transmural.

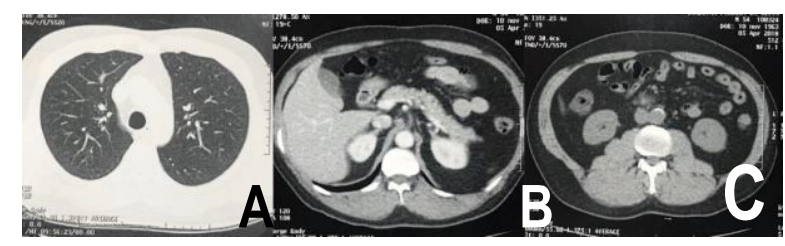

Fonte: Arquivo Pessoal 
Após a confirmação de neoplasia foram solicitados: Tomografia Computadorizada (TC) de Tórax que evidenciou a ausência de derrame pleural e parênquima pulmonar com coeficientes de atenuação normais (Fígura 2); TC de Abdome Total mostrando uma lesão no cólon direito sugestiva de neoplasia com acometimento transmural e fígado, com morfologia, dimensões e coeficiente de atenuação normais.- afastando metástases à distância (Figura 3); e o antígeno carcinoembrionário (CEA) aumentado, com valor igual a 48,47. Caracterizando o estádio B2 de Dukes. O hemograma se mostrava normal, a não ser pela presença de anemia microcítica e hipocrômica.

Foram solicitados exames para avaliação do risco pré operatório, realizou um ecocardiograma que apresentou a seguinte alteração: hipocinesia das regiões apical e média da parede inferior; acinesia da região média da parede septal; e insuficiência mitral de grau discreto. Apresentava também, na coronariografia, cardiopatia obstrutiva com oclusão da artéria marginal esquerda. Desta forma, foi estabelecido risco cardiovascular moderado. Após a avaliação destes exames, a internação para cirurgia foi programada para 01/05.

Figura 4. Ileotransversoanastomose latero-lateral.

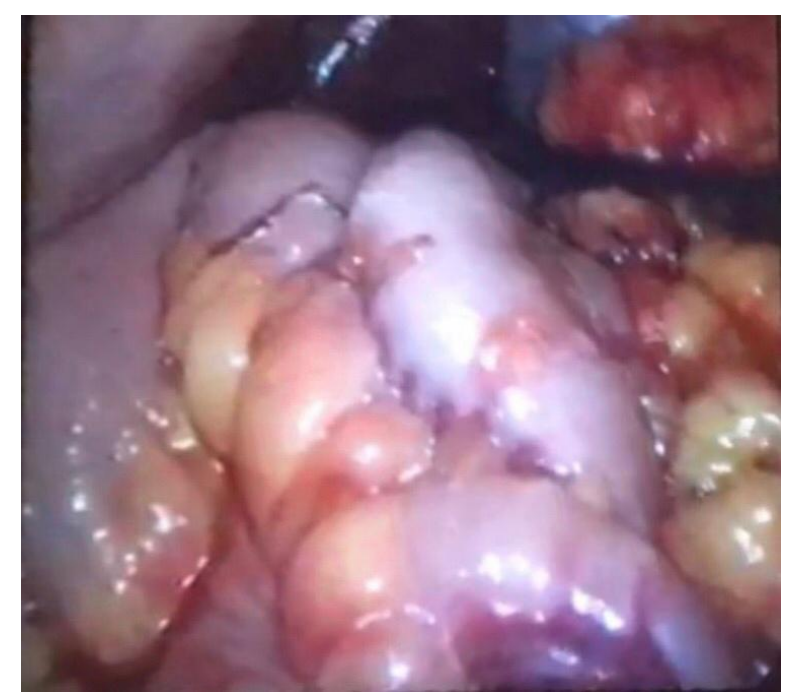

Fonte: Arquivo Pessoal

No dia 02/05, segundo dia de internação hospitalar, foram realizados exames laboratoriais que demostraram anemia microcítica e hipocrômica, leve aumento do lactato e acidose metabólica. Foi instituído dieta zero, antibioticoprofilaxia e realizado o preparo pré operatório. O procedimento escolhido foi hemicolectomia direita laparoscópica com linfadenectomia, ileotransversoanastomose latero-lateral (Figura 4) e drenagem da cavidade, paciente em decúbito dorsal horizontal, sob anestesia geral. Realização de assepsia e 
antissepsia. Pneumoperitônio pela técnica aberta. Inserção de três trocates por visão direta. Inspeção laparoscópica inicial: ausência de metástases hepáticas e para peritônio. Dissecção e ligadura individualizada na artéria e veia cólicas direitas na origem, próximo ao duodeno. Manobra de Catel com liberação do ângulo hepático. Lifadenectomia retroperitoneal com bisturi ultrassonico. Secção ileal e do transverso com endogrampeador.. Incisão transversa para retirada da peça. Ileotransversoanastomese latero lateral em dois planos - sutura manual. Drenagem da cavidade. Revisão da hemostasia. Inspeção laparoscópica final. Síntese nos locais dos portais. Ausência de intercorrências.

A peça cirúrgica (Figura 5) foi encaminhada para a análise anatomopatológica: adenocarcinoma tubular moderadamente diferenciado com infiltração neoplásica até a subserosa e ausência de metástase em 14/14 linfonodos coletados - estádio B2 segundo o estadiamento de Dukes. O paciente teve boa evolução pós-operatória com eliminação de flatos a partir do $2^{\circ}$ dia pós operatório (DPO) e fezes a partir do $3^{\circ}$. Recebe alta no $4^{\circ} \mathrm{DPO}$, assintomático.

Figura 5. Peça cirúrgica

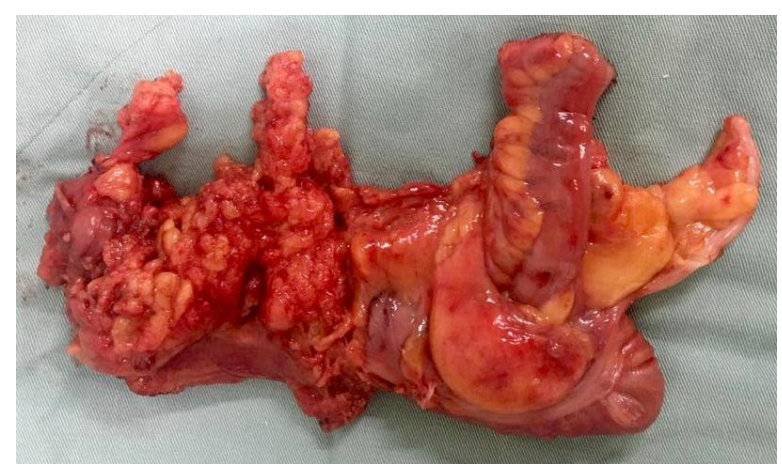

Fonte: Arquivo Pessoal

\section{Discussão}

A neoplasia de cólon representa terceiro lugar em incidência para homens e segundo para mulheres, excluindo-se os tumores de pele. No Brasil, aparece entre os cinco primeiros tipos de câncer mais frequentes (INCA, 2018). O risco deste tipo de neoplasia aumenta com a faixa etária, manifestando-se em mais de 50\% dos casos após 60 anos (Balmagambetova et al., 2020; Shang et al., 2020). No presente relato trata-se de um paciente do sexo masculino com 54 anos de idade, discordando um pouco da literatura. 
Dentre os fatores de risco para neoplasia colorretal encontram-se: idade maior que 60 anos; poliposes hereditárias; síndrome de Lynch; história familiar positiva; doenças inflamatórias intestinais; etilismo; tabagismo; obesidade; sedentarismo; e diabetes (BicudoSalomão et al., 2019). No paciente do caso relatado é diabético de longa data.

Quando se diz respeito às manifestações clínicas, a maior parte dos casos é assintomática, e quando presentes os sintomas se dividem de acordo com sua localização e morfologia. Tumores de colón descendente apresentam alteração do hábito intestinal e sintomas obstrutivos. Anemia ferropriva com fadiga, sangue oculto nas fezes, melena, e sintomas dispépticos são característicos de câncer do cólon ascendente (Norollahi et al., 2020). Conforme descrito no presente relato onde o paciente apresentara apenas um quadro de anemia a esclarecer, oligossintomático devido o diagnóstico da doença ter sido realizado em estágio inicial.

O padrão ouro para investigação de neoplasia colorretal é a colonoscopia que permite o diagnóstico através da biopsia de lesões suspeitas e deve ser realizado em pacientes acima de 50 anos sem fatores de risco (Saraceni et al., 2019; Villanueva-Pájaro et al., 2020). Na colonoscopia realizada no paciente do caso estudado observou-se, no cólon ascendente, uma extensa lesão vegetante, com formato irregular corroborando com a hipótese de neoplasia de cólon direito. A biópsia confirmou o diagnóstico identificando adenocarcinoma tubular moderadamente diferenciado, tipo histológico mais frequente de acordo com a literatura.

Os sítios mais comuns de metástase, em vigência de neoplasia colorretal, são fígado, pulmão, peritônio e linfonodos. Desta forma para estadiamento faz-se necessário a solicitação de tomografias computadorizadas (TC) de tórax e abdome, que foram realizadas no paciente em questão afastando a possibilidade de metástases a distância. Após a confirmação diagnóstica e realização de exames de imagem adequados classificou-se o paciente em estádio B2 de Dukes e estádio IIA de acordo com o TNM (Mohy-ud-din et al., 2019).

Também foi realizada a dosagem do antígeno carcinoembrionário, para estadiamento, que mostrou-se aumentado concordando com a literatura que afirma que $75 \%$ das neoplasias colorretais apresentam a mesma alteração (Diger et al., 2019).

Durante a avaliação pré-operatória foram realizados os seguintes exames: hematócrito, hemoglobina, TC de tórax, ECG, creatinina que não apresentaram alterações e estão de acordo com a literatura (Pacheco-Pérez et al., 2019). Devido a história patológica de infarto agudo do miocárdio e de diabetes melito foram realizados exames complementarem como ecocardiograma e coronariografia que documentaram uma coronariopatia obstrutiva, evidenciando um risco cardíaco intermediário. 
A melhor estratégia terapêutica para o tratamento do câncer em cólon ascendente, pensando em cura do paciente é obtida por meio da cirurgia. Deve ser realizado uma hemicolectomia direita associada a linfadenectomia retroperitoneal, procedimento adotado no caso descrito. A opção por videolaparoscopia mostrou redução do tempo de cirurgia, das alterações metabólicas e traumáticas tendo como consequência uma diminuição da dor no pós-operatório, retorno breve do peristaltismo intestinal, menor risco de infecção transoperatória e redução do tempo de internação, benefícios observados na evolução do paciente (Dominguez \& Bierrenbach, 2020).

De acordo com a literatura deve ser realizado uma linfadenectomia retroperitoneal com, no mínimo, 12 linfonodos e o inventário da cavidade. No paciente em questão, o procedimento foi adequado, afastando a possibilidade de disseminação e descartando acometimento linfonodal em todos os 14 linfonodos coletados (D. Santos et al., 2019).

O benefício da quimioterapia adjuvante está estabelecido para pacientes em fases mais avançadas da doença - Dukes C e estádios III e para estádio II (T3N0M0) quando há instabilidade microssatélite, como o relatado (de Matos et al., 2020) porém a quimioterapia apresenta cardiotoxicidade devido a vasoconstrição sendo contraindicada em paciente coronariopatas o que justifica a não realização desta opção terapêutica na abordagem do paciente (Fayadh et al., 2019; Silva et al., 2020).

O segmento do paciente após o tratamento cirúrgico deve ser realizado com consulta médica e dosagem do CEA a cada 3 a 6 meses por dois anos; colonoscopia após um ano (Amaral et al., 2020)). De maneira geral, a sobrevida em 5 anos para o adenocarcinoma é de 65\%). Atualmente, o paciente relatado está com 6 meses de seguimento sem evidência de doença clínica (Aguiar Junior et al., 2020)).

\section{Considerações finais}

A eficácia e a segurança do procedimento laparoscópico vem sendo demonstrada desde dos anos 90 para o tratamento de patologias colorretais, a técnica já é inclusive utilizada no tratamento cirúrgico oncológico. O paciente em estudo apresentou sinais e sintomas indicativos de neoplasia colorretal, sendo então submetido à colonoscopia, exame que segundo a revisão literária é o mais eficaz e indicado nestes casos. Foi encontrado um achado endoscópico, no cólon ascendente, uma extensa lesão vegetante, com formato irregular, multinodular ou multibocelada, consistência endurecida e fácil sangramento, com dificuldade de progressão do colonoscópio, e confirmação de carcinoma colorretal pela biópsia, eventos 
que levaram a opção pela hemicolectomia direita por via videolaparoscópica. Paciente apresentou evolução satisfatória, recebendo alta hospitalar quatro dias após a realização dos procedimentos citados acima, com uma boa resolução clínica.

Observamos que a paciente foi conduzida de acordo com as preconizações atuais encontradas na literatura para o manejo de cada um dos quadros apresentados, realizando os exames diagnósticos e as intervenções terapêuticas em tempo hábil, o que acreditamos ter sido motivo para o bom desfecho desta apresentação clínica. Nas condições que foram realizados o presente estudo podemos concluir que a via laparoscópica se mostrou como um método eficaz para o tratamento deste paciente portador de neoplasia de cólon ascendente.

Fica como maior sugestão para trabalhos futuros, a pesquisa de dados que melhor quantifiquem o verdadeiro impacto na escolha de videolaparoscopia como procedimento padrão no tratamento do câncer de cólon, no que diz respeito às reduções do tempo de cirurgia, da resposta metabólica ao trauma, da taxa de infecção transoperatória e do tempo de internação. Tais dados ampliará o uso de procedimentos minimamente invasivos para tratamento oncológico, quando disponível, implicando em menor morbimortalidade para os pacientes e, talvez, numa maior sobrevida.

\section{Referências}

Aguiar Junior, S., Oliveira, M. M. de, Silva, D. R. M., Mello, C. A. L. de, Calsavara, V. F., \& Curado, M. P. (2020). Survival of patients with colorectal cancer in a cancer center. Arquivos de Gastroenterologia, 57(2), 172-177.

Amaral, E., Bernardes, M., Ribeiro, S., Rosa, B., Pereira, A., \& Martins, S. F. (2020). Predictors of pathological response and clinical outcome following chemoradiation for locally advanced rectal cancer - a systematic review. Journal of Coloproctology, 40(3), 278-299.

Balmagambetova, S., Bhat, S. A., Koyshybaev, A., Gumarova, Z., Urazayev, O., Tulyaeva, A., Urazova, A., \& Taumova, A. (2020). Trends in colorectal cancer incidence in western Kazakhstan through the first decade of the screening implementation, 2009-2018. Journal of Coloproctology, 40(1), 43-49. 
Bicudo-Salomão, A., Salomão, R. de F., Cuerva, M. P., Martins, M. S., Dock-Nascimento, D. B., \& Aguilar-Nascimento, J. E. de. (2019). Factors related to the reduction of the risk of complications in colorectal surgery within perioperative care recommended by the acerto protocol. ABCD. Arquivos Brasileiros de Cirurgia Digestiva (São Paulo), 32(4), e1477.

Bustamante-Lopez, L. A., Nahas, S. C., Nahas, C. S. R., Pinto, R. A., Marques, C. F. S., \& Cecconello, I. (2019). Is there a difference between right- versus left-sided colon cancers? Does side make any difference in long-term follow-up? ABCD. Arquivos Brasileiros de Cirurgia Digestiva (São Paulo), 32(4), e1479.

D. Santos, M., Silva, C., Oliveira, J., Brandão, P., Sampaio, M., Silva, A. C., Rocha, A., Matos, E., \& Marcos-Pinto, R. (2019). Extensive colectomy in colorectal cancer and hereditary nonpolyposis colorectal cancer - long-term results. Journal of Coloproctology, 39(3), 223-230.

de Matos, M. B., Barbosa, L. E., \& Teixeira, J. P. (2020). Narrative review comparing the epidemiology, characteristics, and survival in sporadic colorectal carcinoma/Lynch syndrome. Journal of Coloproctology, 40(1), 73-78.

Diger, N. R., Kubrusly, L. F., Nassif, P. A. N., Parada, A. A., Bolsi, G. T., Teixeira, H. C. B., \& Malafaia, O. (2019). Is superficial colorectal lesions with low and high grades intraepithelial neoplasms more prevalent in older above 65 years? ABCD. Arquivos Brasileiros de Cirurgia Digestiva (São Paulo), 32(4), e1478.

Dominguez, R. G. S., \& Bierrenbach, A. L. (2020). Hospital morbidity and colorectal cancer mortality: Implications for public health in brazil. Arquivos de Gastroenterologia, 57(2), 182187.

Fayadh, M. H., Sabih, S. A., \& Quadri, H. A. (2019). 8 years observational study on colorectal cancer in UAE. Journal of Coloproctology, 39(4), 394-395.

Instituto Nacional Do Câncer (INCA). Estimativa 2018 Incidência de Câncer no Brasil. Rio de Janeiro: INCA, 2018. 
Mohy-ud-din, N., Umar, S., Syed, A., \& Monga, D. (2019). Adherence to post colorectal cancer surveillance colonoscopy guidelines at a tertiary care center. Journal of Coloproctology, 39(4), 303-308.

Norollahi, S. E., Hamidian, S. M. T., Kohpar, Z. K., Azadi, R., Rostami, P., Vahidi, S., Ghazanfari, S., Shabe, F. A., Khaksar, R., \& Samadani, A. A. (2020). The fluctuation of APC gene in WNT signaling with adenine deletion of adenomatous polyposis coli, is associated in colorectal cancer. Journal of Coloproctology, 40(2), 135-142.

Pacheco-Pérez, L. A., Ruíz-González, K. J., de-la-Torre-Gómez, A. C. G., Guevara-Valtier, M. C., Rodríguez-Puente, L. A., \& Gutiérrez-Valverde, J. M. (2019). Fatores ambientais e conscientização sobre o câncer colorretal em pessoas com risco familiar. Revista LatinoAmericana de Enfermagem, 27, e3195.

Soares Pereira A., Moreira Shitsuka, D., José Parreira, F. \& Shitsuka R.(2018). Metodologia da pesquisa científica. [e-book]. Santa Maria. Ed. UAB/NTE/UFSM. Retrieved from https://repositorio.ufsm.br/bitstream/handle/1/15824/Lic_Computacao_MetodologiaPesquisa-Cientifica.pdf?sequence $=1$.

Saraceni, A. F., Azevedo, R., Almeida, C. M. G., Baraviera, A. C., Kiss, D. R., \& Almeida, M. G. (2019). Association of fecal occult blood tests results with colonoscopic findings in a general hospital and validation of the screening test. Journal of Coloproctology, 39(2), 121126.

Shang, X.-Q., Liu, K.-L., Li, Q., Lao, Y.-Q., Li, N.-S., \& Wu, J. (2020). ADAMTS4 is upregulated in colorectal cancer and could be a useful prognostic indicator of colorectal cancer. Revista da Associação Médica Brasileira, 66(1), 42-47.

Silva, F. M. M. D., Duarte, R. P., Leão, C. C. A., Vissoci, C. M., Alvarenga, A. L. A. T., Ramos, A. B. S., \& Goulart, A. E. C. (2020). Colorectal cancer in patients under age 50: A five-year experience. Revista do Colégio Brasileiro de Cirurgiões, 47, e20202406. 
Villanueva-Pájaro, D. J., Vergara-Dagobeth, E. E., Suárez-Causado, A., \& Gómez-Arias, R.

D. (2020). Epidemiología de la interrelación cáncer colorrectal y diabetes mellitus tipo 2. Revisión sistemática. Revista Facultad Nacional de Salud Pública, 38(2), 1-21.

\title{
Porcentagem de contribuição de cada autor no manuscrito
}

\author{
Marcela Araujo Pereira - 18\% \\ Rebeca Fontoura Morais Abreu - 18\% \\ Fabiana Gallo - $18 \%$ \\ Gabriela Vellano de Andrade - 18\% \\ Rone Antônio Alves de Abreu - 8\% \\ Rone Fontoura Abreu - 12\% \\ Pedro Ernesto Alves Mangueira Júnior - 8\%
}

